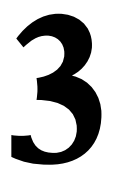

\title{
EVALUACIÓN DEL POTENCIAL EMPRENDEDOR EN ESCOLARES. UNA INVESTIGACIÓN LONGITUDINAL ${ }^{1}$
}

\author{
(ASSESSMENT OF ENTERPRISE POTENTIAL IN STUDENTS. \\ A LONGITUDINAL STUDY)
}

Antonio Bernal Guerrero

Antonio Ramón Cárdenas Gutiérrez

Universidad de Sevilla

DOI: $10.5944 / e d u c X X 1.19032$

\section{Cómo referenciar este artículo/How to reference this article:}

Bernal Guerrero, A.; Cárdenas Gutiérrez, A. R. (2017). Evaluación del potencial emprendedor en escolares. Una investigación longitudinal. Educación XX1, 20(2), 73-94, doi: 10.5944/educXX1.19032

Bernal Guerrero, A. \& Cárdenas Gutiérrez, A. R. (2017). [Assessment of enterprise potential in students. A longitudinal study]. Educación XX1, 20(2), 73-94, doi: 10.5944/educXX1.19032

\section{RESUMEN}

La implantación curricular de la educación emprendedora en la escuela se ha ido produciendo paulatinamente. Algunos programas representan este interés por el fomento de la cultura emprendedora, como EME, EJE e ÍCARO. El éxito de esta educación depende de factores personales, además de ciertos factores externos, que conforman el potencial emprendedor de un sujeto. Nuestra investigación pretende describir el efecto en el potencial emprendedor de un proyecto compuesto por los tres programas mencionados, tratando de comprender los procesos implicados desde la perspectiva del profesorado participante. Realizamos un estudio de casos ex post-facto mediante una investigación descriptivo-longitudinal. El diseño es mixto y exploratorio, siendo el método primario el cualitativo, y secundario el cuantitativo. Los resultados de la técnica cuantitativa se emplean como input para potenciar la técnica cualitativa. Alumnos y profesores han sido seleccionados mediante muestreo intencional. Cuantitativamente, el instrumento utilizado ha sido el Test ATE; y, en la dimensión cualitativa, hemos empleado las entrevistas en profundidad. Para evaluar el efecto del proyecto, empleamos un análisis de la varianza mediante un diseño factorial $2 \times 2$ con medidas repetidas. Los datos recabados se analizaron mediante un ANOVA mixto a través del programa estadístico SPSS versión 2.0. Para tratar la información obtenida mediante las entrevistas, realizamos un análisis semiótico y narrativo. El efecto de los programas en el potencial emprendedor se ha mostrado 
prácticamente nulo. La frecuencia participativa tampoco ha tenido un efecto apreciable. El análisis y discusión de la información obtenida parece indicar que la implementación de los programas debe realizarse de modo que se incluyan pertinente y satisfactoriamente los factores personales relativos al desarrollo del potencial emprendedor.

\section{PALABRAS CLAVE}

Educación emprendedora; formación empresarial; programa de formación; enseñanza obligatoria; formación de profesores.

\section{ABSTRACT}

The curricular implementation of entrepreneurship education in schools has been occurring gradually. Some programs represent this interest in the promotion of entrepreneurial culture, such as EME, EJE and ÍCARO. The success of this education depends on personal factors, in addition to certain external factors, which make up the enterprise potential of a subject. Our research aims to describe the effect on the enterprise potential of a project consisting of the three programs mentioned, as well as to understand the processes involved from the perspective of the teachers taking part. We conducted a study of ex post-facto cases via longitudinal descriptive research. The design is mixed and exploratory, the primary method being qualitative and the secondary quantitative. The results of the quantitative technique are used as an input to enhance the qualitative technique. The students and teachers have been selected through purposive sampling. Quantitatively, the instrument used was the ATE Test; and in the qualitative dimension, we have used in-depth interviews. To evaluate the effect of the project, we used an analysis of variance via repeated measures of a 2 x 2 factorial design. The collected data were analyzed using a mixed ANOVA through the statistical program SPPS version 20 . To process the information obtained from the interviews, we performed a semiotic and narrative analysis. The effect of the programs on enterprise potential has been virtually non-existent. The participatory frequency has not had an appreciable effect. The analysis and discussion of the information obtained seems to indicate that the implementation of the programs that must be carried out will include relevant and successful personal factors relating to the development of the enterprise potential.

\section{KEY WORDS}

Entrepreneurship education; management education; training programme; compulsory education; teacher education. 


\section{INTRODUCCIÓN}

Dado el enfoque predominantemente socioeconómico que presenta el impulso del emprendimiento en el contexto europeo («entrepreneurship»/«espíritu emprendedor»), no resultará extraña la identificación frecuente del potencial emprendedor con la exclusiva capacidad de generar empresa y desarrollar actividad empresarial (Habiby, Deirdre y Coyle, 2010; González y Zúñiga, 2011). No obstante, el potencial emprendedor puede considerarse, desde la perspectiva educacional, como el cúmulo de capacidades que un sujeto puede desarrollar configurando su identidad emprendedora. Dichas capacidades emprendedoras, en sentido estricto, están relacionadas con la dimensión personal del sujeto; aunque puedan presentar obviamente vínculos con el denominado emprendimiento, esto es, con la dimensión abiertamente productiva del proceso educativo. Emergen así diferentes variables de la dimensión personal como potencial emprendedor. Parece evidente su relación con el incremento de la capacidad productiva del sujeto y con su proyección emprendedora en el ámbito socioeconómico y laboral. Por esta razón, observar cómo cambian estas variables en los proyectos aplicados de educación emprendedora, adquiere interés para el diagnóstico renovado de los mismos y para la orientación de la posterior toma de decisiones.

El potencial emprendedor se halla íntimamente relacionado con determinadas cualidades personales como la creatividad, el espíritu innovador, el sentido crítico, el control personal, la responsabilidad, la autoconfianza, la motivación de logro, la perseverancia, la disposición para la resolución de problemas, el liderazgo o la resistencia al fracaso, entre otras. No resulta extraño, por tanto, que se haya articulado el contenido del potencial emprendedor en torno a determinadas cualidades personales. Lumpkin y Dess (1996), al referirse a la orientación emprendedora del individuo, estructuraron cinco dimensiones: autonomía, innovación, tolerancia al riesgo, proactividad y competitividad. Recientemente (Bernal y Cárdenas, 2014), señalamos varios indicadores personales significativos (autonomía, iniciativa, creatividad y espíritu cooperativo). Athayde (2009) ha sintetizado el potencial emprendedor en cinco variables (creatividad, control personal, orientación al logro, resolución de problemas y liderazgo). Así, en la medida en que podemos hablar de una identidad (personalidad) emprendedora construida socialmente (Chell, 2008), pasa a un primer plano la investigación centrada en el valor de las actitudes y cualidades personales vinculadas a su desarrollo (Liñán, Santos y Fernández, 2011). El Programa Educación y Formación 2010 de la Comisión Europea, al definir el «espíritu emprendedor», enfatiza las cualidades más personales sobre aquellas más propias de la iniciativa empresarial. Realmente, no puede restringirse la educación emprendedora al ámbito del conocimiento que se ha categorizado como 
«business», puesto que se trata de un concepto más amplio, más profundo y más rico, alcanzando en última instancia al desarrollo de la autonomía y la iniciativa personal y a la concepción misma de la ciudadanía y la responsabilidad cívica (Jones e Iredale, 2010).

Desde la asunción de esta complejidad conceptual del potencial emprendedor, pero en última instancia vinculada al desarrollo del emprendimiento económico, se ha insistido en la formación de la competencia de emprender, incardinada en el enfoque de formación en competencias (COM, 2012). En el caso español, el Ministerio de Industria, Comercio y Turismo (2010) ha seleccionado las políticas y prácticas más relevantes sobre actividades emprendedoras desarrolladas en nuestro país. Entre estas, existen programas que tratan de fomentar el emprendimiento en los escolares y jóvenes españoles. Entre ellos, EME (Emprender en Mi Escuela) y EJE (Empresa Joven Europea), creados por Valnalón en Asturias e implantados en otras Comunidades Autónomas, dirigidos, respectivamente, a la Educación Primaria y a la Educación Secundaria Obligatoria, bajo la fórmula de la sociedad cooperativa, tienen como propósito que los escolares aprendan a crear y gestionar sus empresas cooperativas, a presentarlas al público y a vender los artículos elaborados, tratando de aproximar la empresa al aula. Junto a ellos, el programa ÍCARO en Andalucía, participa de los mismos propósitos, aunque vincula su actividad al sector creativo-cultural, mediante las cooperativas de servicios. Asociados al espíritu emprendedor, estos programas han venido fraguando una idea de emprendimiento que contiene determinados valores como la creatividad, la autonomía y la responsabilidad, o el espíritu de equipo y el liderazgo. Concretamente, nuestra investigación gira en torno a un proyecto de educación emprendedora que ha aunado estos tres programas.

\section{PLANTEAMIENTO DEL PROBLEMA Y OBJETIVOS}

La educación es un elemento clave para desarrollar en la juventud las actitudes y habilidades precisas. Parece importante que la educación emprendedora comience a edades tempranas (EACEA, 2012). Sin embargo, la mayor parte de la educación e investigación teórico-práctica sobre el emprendimiento se ha centrado en el ámbito universitario (Vesa y Down, 2012; Rae y Woodier, 2012), con exigua repercusión en los niveles educativos inferiores. El emprendimiento como ámbito de investigación en las escuelas es reciente (Draycott, Rae y Vause, 2011). Los estudios que relacionan emprendimiento y escuela escasean, centrándose estos trabajos en los resultados del aprendizaje, la conceptualización de la educación emprendedora o la evaluación de los programas de emprendimiento en las escuelas. Por otra parte, parece que la perspectiva del profesorado no ha 
sido suficientemente atendida (Jones e Iredale, 2010), aun siendo crucial el rol que desempeñan los docentes en la implementación de la educación emprendedora (Ruskovaara y Pihkala, 2013). Quizás, la perspectiva del profesorado podría iluminar un poco más la «caja negra» de los programas de educación emprendedora (PEE) a la que hacen referencia Maritz y Brown (2013). Por lo demás, hay una carencia evidente de estudios de corte longitudinal que puedan contribuir, desde su singular riqueza (Chávez, 2012; Gewerc y Montero, 2013), al progreso de su conocimiento y a su avance teórico y práctico.

Se precisaría explorar, consiguientemente, desde una perspectiva temporal sólida, cómo se están desarrollando los PEE (principios, objetivos, métodos, contenidos y resultados) según los docentes implicados en su implementación (Louise-Jayne y Muir, 2012).

Como línea rectora de la investigación, con respecto a la implementación y desde la óptica del profesorado, estudiamos con carácter descriptivo y exploratorio el desarrollo de los programas que constituyen el proyecto de educación emprendedora, para tratar de comprender su sentido y alcance respecto del potencial emprendedor de los sujetos educandos, desarrollado a lo largo de cinco años.

Atendiendo al interés en comprender el desarrollo de la educación emprendedora en la educación obligatoria, desde la perspectiva del profesorado, y en su repercusión en la dimensión emprendedora de la identidad personal, los objetivos que perseguimos son: 1) describir desde una perspectiva longitudinal, el efecto de un proyecto de educación emprendedora, formado por los programas EME, ÍCARO y EJE, sobre la identidad (potencial emprendedor) del alumnado; 2) comprender los procesos de enseñanza y aprendizaje que se han implementado en dicho proyecto a lo largo del tiempo durante las etapas de Educación Primaria y ESO, vertebrándolos en sus principales dimensiones curriculares desde un enfoque emprendedor.

\section{MÉTODO}

Es un estudio de casos (Yin, 2009) ex post-facto realizado mediante una investigación descriptivo-longitudinal de medidas repetidas antes y después a dos cohortes de escolares (mediante la aplicación del test ATE), junto a tres entrevistas (inicial, media y final: EI, EM y EF) realizadas a los docentes responsables del proyecto (Arnau, 1995; Bisquerra, 2012). El diseño y método de investigación es mixto y exploratorio (Heyvaert, Hannes, Maes y Onghena, 2013). Teniendo presente las decisiones de prioridad 
y secuencia del método (Morgan, 1998), decidimos que el método primario sea el cualitativo (QL), siendo secundario el cuantitativo (qt); y que la secuencia temporal sea cuantitativa y cualitativa. Ambas técnicas se integran mediante la estrategia de combinación; esto es, los resultados de la técnica cuantitativa se emplean como input para potenciar la técnica cualitativa, cuyo output configura el objetivo de la investigación (Bericat, 1998). Los resultados del pre-test y del post-test han sido utilizados, respectivamente, como input de información en el desarrollo de las entrevistas iniciales y finales al profesorado, siendo las narraciones de los docentes, obtenidas en las entrevistas, el output de la investigación. Asumiendo la complejidad de integrar métodos de investigación cualitativos y cuantitativos (Mayoh y Onwuegbuzie, 2015), elaboramos un diseño multifase, resultando el siguiente protocolo en función de la alternancia metódica: qtàQLàQLà qtàQL. La validez del estudio se realiza mediante una triangulación de tiempo (Pérez Serrano, 1994), analizando los cambios producidos en los tres momentos de medida, y una triangulación inter-método combinando métodos cuantitativos y cualitativos (Denzin, 1970).

\section{MUESTRA}

Alumnos y profesores de un centro educativo andaluz (donde se ha aplicado el proyecto de educación emprendedora mencionado: EME, para el último ciclo de Educación Primaria; ÍCARO y EJE, para el primer y segundo ciclo de la ESO), fueron seleccionados mediante un muestreo intencional (Ruiz, 2009). Los criterios estratégicos fueron: accesibilidad al campo de estudio y conocimiento previo de los informantes. Los sujetos entrevistados fueron los cinco profesores que implementaron los programas, con experiencia docente media de 20 años y con la condición de que participaban por primera vez en este proyecto. La muestra inicial estaba formada por un total de 57 alumnos matriculados durante el curso 2008/2009, de 5. ${ }^{\circ}$ y $6 .^{\circ}$ de Educación Primaria, y que participaron sucesivamente hasta el curso 2012/2013. Sin embargo, debido a la mortandad de 5 sujetos por cambio de centro, la muestra final se redujo a 52 (50\% mujeres y $50 \%$ varones). Aunque en principio el estudio lo conformaría un único grupo, la baja por enfermedad de un profesor del primer ciclo de la ESO (P3, véase Tabla 1) propició que la cohorte 2 no participara en el programa ÍCARO durante los cursos 2010-11 y 2011-12; así, la muestra quedó definitivamente dividida en dos cohortes bajo el criterio del número de participaciones en los programas. En el primer pre-test, los alumnos presentaban las siguientes edades: un 46,1 \%,10 años; un 48,1\%, 11; y un $5,8 \%, 12$. El 42,3\% pertenecía a la cohorte 1 (6. ${ }^{\circ}$ Educación Primaria) y el $57,7 \%$ a la cohorte 2 (5. ${ }^{\circ}$ Educación Primaria). En el post-test, un 38,5 \% tiene 14 años; un 34,6\%, 15; y un 26,9\%, 16. Por las repeticiones, el 2,3\% 
de la cohorte 1 pasó a la cohorte 2 , quedando configuradas respectivamente por las siguientes frecuencias: 22 (C1) y 30 (C2).

\section{INSTRUMENTOS}

La información cuantitativa se recogió mediante el Test ATE (Athayde, 2009), que mide el potencial o actitud emprendedora. Su validez y fiabilidad ha sido contrastada (Steenekamp, van der Merwe y Athayde, 2011; Athayde, 2012). Con un total de 30 ítems con 7 opciones de respuesta tipo Likert, está estructurado en 5 subescalas referentes a las dimensiones del potencial emprendedor, junto con la puntuación total del constructo:

- Creatividad: capacidad de generar ideas facilitadoras del proceso innovador.

- Control personal: capacidad para manejar y autorregular los comportamientos propios.

- Orientación al logro: capacidad de perseverancia y de proactividad hacia la consecución de metas.

- Resolución de problemas: capacidad para afrontar circunstancias de incertidumbre e intensidad.

- Liderazgo: capacidad de construir equipos de trabajo, tomar decisiones, negociar y planificar.

Cualitativamente, utilizamos la entrevista en profundidad, semiestructurada (Kvale, 2011). Configurada internamente por tres bloques temáticos: Fundamentación y finalidades del proyecto, contenidos y metodología, y evaluación de los programas. Esta estructura sirvió para los tres momentos de medida, añadiendo en la última los resultados cuantitativos del alumnado. Utilizamos claves de identificación para clasificar las entrevistas (p.e.: EMP1C16P, quiere decir: E: entrevista inicial, media o final; P: identificación numérica de cada profesor; C: cohorte 1 o $2, \mathrm{~N} .^{\circ}$ : curso; letra mayúscula, P-E: etapa educativa Primaria o ESO). Los bloques se redactaron a partir de indicadores de evaluación de programas educativos y de la competencia básica próxima a la autonomía e iniciativa personal (Casanova, 2012; Roca 2013). La estructura final se elaboró a partir de las recomendaciones de una selección intencionada de cinco jueces expertos (Banister, Burman, Parker, Taylor y Tindall, 2004). Para controlar los elementos espurios contemplamos los siguientes criterios (Gibbs, 2012; Bisquerra, 2012): credibilidad, mediante la comprobación de los relatos (contraste de la información narrada 
en las entrevistas iniciales y medias con las entrevistas finales) y la triangulación de tiempo e intermétodo; transferibilidad, mediante muestreo teórico (muestreo intencional), descripción minuciosa de informantes y recogida de datos; dependencia, mediante las claves de identificación y descripción de las técnicas de análisis de datos; y confirmabilidad, por análisis de datos a través del programa atlas.ti. V7.0 y comprobación de la información con los entrevistados.

\section{PROCEDIMIENTO}

Consideramos tres fases de recogida de datos, donde se han utilizado técnicas cuantitativas, vinculadas al alumnado (test ATE), y cualitativas, ligadas al profesorado (entrevistas), durante cinco cursos académicos (Tabla 1):

- Fase $1 .^{a}$ (2008-2009 y 2009-2010): recogida de información cuantitativa proporcionada por los alumnos de $6 .^{\circ}$ (cohorte 1 : C1) y $5 .^{\circ}$ (cohorte 2: C2) de Educación Primaria, junto con los datos cualitativos facilitados por los cinco profesores en las entrevistas iniciales (EI).

- Fase 2. ${ }^{a}$ (2010-2011 y 2011-12): recogida de datos cualitativos de cuatro profesores (uno no participó) mediante las entrevistas medias (EM).

- Fase $3 .^{a}(2012-13)$ : a) cuantitativa: la relativa a la cohorte $2\left(3 .^{\circ}\right.$ ESO) y la correspondiente a la cohorte 1 ( $4 .^{\circ}$ ESO); b) cualitativa: mediante cinco entrevistas finales (EF) a los profesores, una vez analizados los datos cuantitativos.

Tabla 1

Estructura de recogida longitudinal de datos

\begin{tabular}{|c|c|c|c|c|c|c|c|c|c|c|}
\hline \multirow{3}{*}{$\begin{array}{c}\text { Fases } \\
\begin{array}{c}\text { Cursos } \\
\text { Académicos }\end{array} \\
\text { Cohortes }\end{array}$} & \multicolumn{4}{|c|}{ Fase $1 .^{\mathrm{a}}$} & \multicolumn{4}{|c|}{ Fase $2 .^{\mathrm{a}}$} & \multirow{2}{*}{\multicolumn{2}{|c|}{$\begin{array}{c}\text { Fase } 3 .^{a} \\
2012-13\end{array}$}} \\
\hline & \multicolumn{2}{|c|}{ 2008-09 } & \multicolumn{2}{|c|}{$2009-10$} & \multicolumn{2}{|c|}{ 2010-11 } & \multicolumn{2}{|c|}{ 2011-12 } & & \\
\hline & C2 & $\mathrm{C} 1$ & $\mathrm{C} 2$ & $\mathrm{C} 1$ & $\mathrm{C} 2$ & $\mathrm{C} 1$ & $\mathrm{C} 2$ & $\mathrm{C} 1$ & $\mathrm{C} 2$ & $\mathrm{C} 1$ \\
\hline Nivel de Curso & 5. ${ }^{\circ} \mathrm{EP}$ & 6. ${ }^{\circ} \mathrm{EP}$ & 6. ${ }^{\circ} \mathrm{EP}$ & 1. ${ }^{\circ} \mathrm{ESO}$ & $1 .^{\circ} \mathrm{ESO}$ & $2 .^{\circ} \mathrm{ESO}$ & 2. ${ }^{\circ} \mathrm{ESO}$ & 3. ${ }^{\circ} \mathrm{ESO}$ & 3. ${ }^{\circ} \mathrm{ESO}$ & 4. ${ }^{\circ} \mathrm{ESO}$ \\
\hline Profesorado & $\mathrm{P} 1$ & P1 & P1 & P2 & P3 & P2 & P3 & P4 & P5 & P4 \\
\hline Programas & EME & EME & EME & ÍCARO & & ÍCARO & & EJE & EJE & EJE \\
\hline $\begin{array}{l}\text { Técnicas } \\
\text { cuantitativas }\end{array}$ & $\begin{array}{r}\text { Pre-te } \\
\text { (c }\end{array}$ & $\begin{array}{l}\text { t ATE } \\
\text { t) }\end{array}$ & & & & & & & $\begin{array}{r}\text { Post-te } \\
\text { ( }\end{array}$ & $\begin{array}{l}\text { st ATE } \\
\text { t) }\end{array}$ \\
\hline $\begin{array}{l}\text { Técnicas } \\
\text { cualitativas }\end{array}$ & $\begin{array}{r}\text { Entre } \\
\text { Inici } \\
5 \text { tu } \\
(0 \\
\end{array}$ & $\begin{array}{l}\text { vistas } \\
\text { les a } \\
\text { ores } \\
\text { L) } \\
\end{array}$ & & & $\begin{array}{r}\text { Entre } \\
\text { Med } \\
4 \text { tut } \\
(\mathrm{Q} \\
\end{array}$ & $\begin{array}{l}\text { vistas } \\
\text { ias a } \\
\text { ores } \\
\text { L) } \\
\end{array}$ & & & $\begin{array}{r}\text { Entre } \\
\text { Fina } \\
5 \text { tut } \\
(\mathrm{Q} \\
\end{array}$ & $\begin{array}{l}\text { vistas } \\
\text { es a } \\
\text { ores } \\
\text { L) }\end{array}$ \\
\hline
\end{tabular}




\section{ANÁLISIS DE DATOS}

Efectuado mediante procedimientos cuantitativos y cualitativos. Operativamente, en la dimensión cuantitativa de la investigación, nos planteamos las siguientes hipótesis: H1) La aplicación de los programas EME, ÍCARO y EJE incrementa el nivel de las variables del potencial emprendedor. H2) El efecto de estos programas aumenta con el número de participaciones del alumnado.

Para evaluar el efecto se empleó un análisis de la varianza mediante un diseño factorial $2 \times 2$ con medidas repetidas, por la ventaja de permitirnos el estudio del efecto de la interacción entre factores (Pardo, Garrido, Ruiz y San Martín, 2007), donde el factor intergrupos es la participación en los programas de emprendimiento, tomando en este caso los niveles de mayor participación $(\mathrm{C} 1+\mathrm{P})$ y menor participación $(\mathrm{C} 2-\mathrm{P})$ en los programas de emprendimiento; y siendo el factor intragrupo la medida repetida del tiempo transcurrido, teniendo como niveles los dos momentos de medida (pretest y postest). En relación a las fuentes de variación intersujeto se comprobó el supuesto de homoestacidad con la prueba de Levene y con respecto a las fuentes de variación intrasujeto se asumió el supuesto de esfericidad con la prueba de Mauchly (Balluerka y Vergara, 2002). Los datos recabados se analizaron mediante un ANOVA mixto a través del programa estadístico SPSS versión 20. Las variables dependientes son las subescalas y puntuación total del ATE y las independientes los programas y la mayor o menor participación en ellos.

En las técnicas cualitativas, bajo un enfoque categórico del contenido (Lieblich, Tuval-Mashiach y Zilber, 1998), se realizó un análisis semiótico y narrativo (León y Montero, 2003) mediante la codificación abierta e inductiva de las narraciones del profesorado, consiguiendo un árbol de categorías emergente que paulatinamente se fue rediseñando (Tabla 2) (Gibbs, 2012). Las narraciones categorizadas se analizaron mediante una matriz descriptiva dicotómica de ausencia-presencia de los ámbitos evaluados, elaborada ad hoc (Tójar, 2006). La información de los docentes se clasificó como ausencia y presencia (dividida en presencia con y sin congruencia interna), lo cual permitió cuantificar las valoraciones del profesorado. 
Tabla 2

Estructura del árbol de categorías, extraída de la narración de los docentes

\begin{tabular}{|c|c|c|}
\hline \multicolumn{3}{|c|}{ ÁRBOL DE CATEGORÍAS } \\
\hline METACATEGORÍAS & CATEGORÍAS & SUBCATEGORÍAS \\
\hline \multirow{2}{*}{$\begin{array}{l}\text { Fundamentación } \\
\text { del proyecto }\end{array}$} & Principios Pedagógicos & $\begin{array}{l}\text { Carencia de base pedagógica, psi- } \\
\text { cológica, social o científica }\end{array}$ \\
\hline & Recursos educativos & $\begin{array}{l}\text { Recursos didácticos on-line } \\
\text { Recursos personales (docentes) }\end{array}$ \\
\hline \multirow[t]{2}{*}{$\begin{array}{l}\text { Finalidades } \\
\text { del proyecto }\end{array}$} & $\begin{array}{l}\text { Objetivos específicos empresaria- } \\
\text { les en EME, EJE e ÍCARO }\end{array}$ & $\begin{array}{l}\text { Creación de cooperativas } \\
\text { Elección de órganos rectores } \\
\text { Plan de fabricación } \\
\text { Constitución de grupos de trabajo }\end{array}$ \\
\hline & $\begin{array}{l}\text { Objetivos específicos personales } \\
\text { en EME, EJE e ÍCARO }\end{array}$ & $\begin{array}{l}\text { Resolución de conflictos } \\
\text { Desarrollo de la responsabilidad }\end{array}$ \\
\hline \multirow{3}{*}{ Contenidos } & Actitudinales & $\begin{array}{l}\text { Habilidades de Comunicación } \\
\text { Habilidades Sociales }\end{array}$ \\
\hline & Procedimentales & Gestión empresarial-cooperativas \\
\hline & Conceptuales & $\begin{array}{l}\text { Conocimiento empresarial-coope- } \\
\text { rativas }\end{array}$ \\
\hline \multirow[b]{2}{*}{ Metodología } & Tipología & $\begin{array}{l}\text { Trabajo por proyectos } \\
\text { Trabajo cooperativo }\end{array}$ \\
\hline & Limitaciones metodológicas & $\begin{array}{l}\text { Falta de dinámicas } \\
\text { Excesivo trabajo cooperativo } \\
\text { Carencia de métodos de activación } \\
\text { motivacional }\end{array}$ \\
\hline \multirow{3}{*}{ Evaluación } & Diseño y planificación & Carencia de pruebas de evaluación \\
\hline & Generación de resultados & \begin{tabular}{|l|} 
Resultados medios \\
Resultados finales
\end{tabular} \\
\hline & Valoración general del proyecto & $\begin{array}{l}\text { Indicadores empresariales } \\
\text { Indicadores personales }\end{array}$ \\
\hline
\end{tabular}

\section{PRESENTACIÓN Y DISCUSIÓN DE RESULTADOS}

\section{Dimensión cuantitativa}

En la Tabla 3 se presentan los resultados del ANOVA mixto, donde no existen efectos significativos del factor entregrupos $(\mathrm{C} 1+\mathrm{P}$ y $\mathrm{C} 2-\mathrm{P})$ sobre las variables dependientes, excepto del logro $\left(\mathrm{F}_{(1,50)}=12.299, \mathrm{p}=.001\right)$; lo que indica la existencia de diferencias en las medias entre la $\mathrm{C} 1+\mathrm{P}$ y la $\mathrm{C} 2-\mathrm{P}$, en la evaluación inicial y final del proyecto. Los resultados desvelan que existe un efecto significativo del factor intragrupo sobre las variables dependientes de creatividad $\left(\mathrm{F}_{(1,50)}=9,860, \mathrm{p}=.003\right)$, liderazgo $\left(\mathrm{F}_{(1,50)}=5,690, \mathrm{p}=.021\right)$, orientación al logro $\left(\mathrm{F}_{(1,50)}=35,977, \mathrm{p}=.000\right) \mathrm{y}$ en las puntuaciones globales del test ATE $\left(\mathrm{F}_{(1,50)}=\right.$ $15,092, \mathrm{p}=.000)$, todas ellas con un tamaño de efecto grande (Etap2 >.14), 
excepto liderazgo con efecto medio $(.06<$ Etap $<.14)$, según se aplica la regla de Cohen (1988). La puntuación de las C1+P y C2-P cambia significativamente con el tiempo en estas variables, según la participación de los alumnos. Para saber si esta transformación se produce de manera similar en las $\mathrm{C} 1+\mathrm{P}$ y C2-P o de forma diferente, observamos el efecto de interacción. Los contrastes de grupo por momento de medida nos desvelan que únicamente es significativo el efecto de interacción en la variable de orientación al logro con un tamaño de efecto medio $\left(\mathrm{F}_{(1,50)}=7,483, \mathrm{p}=.009\right.$; Etap2 = .13). Por el contrario, no se produce efecto de interacción en las variables de creatividad, liderazgo, resolución de problemas, control personal y puntuación global del test ATE. En este caso, los resultados indicarían que EME, EJE e ÍCARO no han incrementado, en general, el nivel de las variables del potencial emprendedor. Por otro lado, el efecto de los programas no incide significativamente en función del número de participaciones del alumnado. Pareciera que el desarrollo completo de una actitud emprendedora se encuentra alejado de los niveles deseados.

Tabla 3

Resultados del análisis factorial de la varianza

\begin{tabular}{|c|c|c|c|c|c|c|c|c|c|}
\hline \multirow[b]{2}{*}{ Variables } & \multicolumn{3}{|c|}{ Efecto Entregrupos } & \multicolumn{3}{|c|}{ Efecto Intragrupo } & \multicolumn{3}{|c|}{ Efecto Interacción } \\
\hline & $\mathbf{F}$ & $\mathbf{P}$ & $\eta \mathbf{p} 2$ & $\mathbf{F}$ & $\mathbf{P}$ & $\eta p 2$ & $\mathbf{F}$ & $\mathbf{P}$ & $\eta \mathbf{p} 2$ \\
\hline Creatividad & 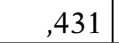 &, 514 & ,009 & 9,860 &, $003^{*}$ &, $165 *$ & 3,221 & ,079 &, 061 \\
\hline Liderazgo & 688 & 411 &, 014 & 5,690 &, $021 *$ &, $102 *$ &, 812 & 372 &, 016 \\
\hline $\begin{array}{l}\text { Resolución } \\
\text { de Problemas }\end{array}$ &, 039 &, 843 & ,001 &, 027 &, 871 &, 001 &, 010 & ,922 &, 000 \\
\hline Orientación Logro & \begin{tabular}{|l|}
12,299 \\
\end{tabular} & ,001* & .197 & 35,977 & ,000* & $418^{*}$ & 7,483 & ,009* &, $130 *$ \\
\hline Control Personal & \begin{tabular}{|l|}
, 161 \\
\end{tabular} &, 690 & ,003 &, 000 & ,998 &, 000 &, 057 &, 813 &, 001 \\
\hline Puntuación ATE & ,436 & ,512 & ,009 & 15,092 &, $000 *$ & $232 *$ & 3,890 & ,054 & ,072 \\
\hline
\end{tabular}

*= significación estadística

\section{Dimensión cualitativa}

\section{Fundamentación del proyecto}

Todos los profesores en los tres momentos de medida coinciden en que el proyecto no presenta una base pedagógica, psicológica, social o científica que lo cimente sólidamente. Al respecto, desde 2012 existe un incipiente conjunto de recursos educativos para los tres programas. Según comenta el tutor de EME, ha habido unos primeros pasos, todavía lejos de lo deseable:

EMP1C16P: «es una simple guía sobre los pasos a seguir para desarrollar EME, aunque es útil aún falta mucho por hacer». 
Igualmente, en las EI, los profesores que han implementado EJE e ÍCARO, comentan no tener recursos educativos, aunque en las EM se vislumbró que institucionalmente se diseñaban recursos didácticos sobre creatividad o habilidades de comunicación. Sin embargo, en las EF se narra que esos materiales solo son presentaciones on-line carentes de material educativo para utilizarlas adecuadamente con los alumnos.

En el proyecto no parece haber directrices de buenas prácticas sobre cómo implementar la educación emprendedora. Fayolle y Gailly (2008) han denunciado asimismo esta carencia en este tipo de proyectos, agravada por el hecho de que los PEE basados en determinadas teorías o modelos de enseñanza son escasamente utilizados.

\section{Finalidades}

En las EI, el profesorado reconoce que no existían finalidades explícitas y únicamente se plantearon objetivos empresariales relacionados con el desempeño de las cooperativas. En las EM y EF los profesores indican haber continuado formulando objetivos de carácter empresarial sobre la ejecución de tareas y el cumplimiento de plazos, pero no ligados al emprendimiento en profundidad, como manifiesta este testimonio:

EMP5C24E: «no nos planteamos objetivos relacionados con la innovación, la creatividad o el liderazgo; es difícil poder formularlos».

Únicamente, en las EF finales el profesorado reconoce que implícitamente se han planteado objetivos relacionados con el ámbito personal: conflictos entre iguales y responsabilidad en el desempeño de las tareas del alumnado.

Aunque, Seikkula, Ruskovaara, Ikavalko, Mattila y Rytkola (2010) plantean que los profesores son fundamentales en la formulación y realización de los objetivos de la educación emprendedora, no sería conveniente delegar toda su formulación en su buen desempeño, especialmente, si se carece de una correcta formación del profesorado relacionada con la práctica de la educación emprendedora. No olvidemos que hay diversidad de elementos implicados en función de la tipología práctica adoptada (Nabi y Liñán, 2011).

\section{Contenidos}

El profesorado comenta en las entrevistas que el proyecto carece de contenidos propios plasmados en unos materiales educativos para el profesorado o para el alumnado. 
EMP2C12E: "No tenemos un contenido propiamente dicho (...) se trata de que los escolares formen cooperativas. Posteriormente, les decimos cuáles son los órganos de gestión, los eligen y, a partir de ahí, a fabricar materiales».

Actitudes, valores o motivaciones, habilidades y conocimientos constituyen los contenidos de la educación emprendedora (Johannison, 1991). Parece que en el proyecto se ha insistido exclusivamente en aquellos contenidos relativos al desarrollo de las cooperativas. La referencia a las habilidades sociales y de comunicación facilitadoras del trabajo cooperativo y de la responsabilidad con los compromisos adquiridos, ha aparecido en tres narraciones en las EM y en todas en las EF, mientras que no surgió en las EI. Así, se desdibujan los indicadores personales (Alemany, Marina y Pérez 2013), tan relevantes para el desarrollo de la identidad emprendedora (Harmeling, 2011).

Por lo demás, como sugieren Draycott y Rae (2011), sin unos contenidos explícitos, el desarrollo de la competencia emprendedora se restringe, pues carece usualmente de una equilibrada articulación de los distintos «saberes» sobre el emprendimiento, como parece haber sucedido en este proyecto.

\section{Metodología}

Del conjunto de entrevistas se deduce que los docentes han pasado de una actitud inicial de control y supervisión constante del alumnado hacia una concepción más facilitadora y mediadora del aprendizaje, mediante metodologías activas, como el aprendizaje cooperativo (Johnson, Johnson y Holubec, 1994) y el trabajo por proyectos (Railsback, 2002). Sin embargo, la inexperiencia inicial del profesorado en la implementación de los programas, junto con la carencia de orientaciones metodológicas, nos hace pensar en la necesidad de una mayor congruencia metodológica (Pérez Juste, 2006) para el desarrollo de estos programas. Esta carencia metodológica en los procesos de enseñanza de la educación emprendedora hace que aparezcan las principales limitaciones metodológicas del proyecto (ausencia de dinámicas preparatorias, excesivo cooperativismo, falta de motivación):

EFP1C16P: "Cada año tienen menos ganas de participar (...) el dato objetivo está en que comparando el primero con los siguientes, cada vez obtienen menos beneficio en las ventas; incluso a veces no cubren el capital social y parece darles igual».

Se advierte, con todo, una progresiva conciencia de la importancia de la metodología en el profesorado participante, fenómeno ya contemplado en otros estudios (Fayolle y Gailly, 2008). 


\section{Evaluación}

En todas las entrevistas aparece un consenso explícito sobre la inexistencia de los elementos constitutivos del proceso evaluativo y de su adecuada planificación. No existe una valoración objetiva del desarrollo de estos programas y tampoco la administración educativa ha favorecido una evaluación de resultados. Los profesores no han tenido información evaluativa sobre el desarrollo del proyecto y su valoración general del mismo ha sido poco favorable.

Con respecto al dominio empresarial, los conocimientos conceptuales y de gestión de la empresarialidad no se han mostrado muy sólidos. En relación con el dominio personal, el profesorado ha reaccionado sin estupor al comprobar la nula significatividad en los indicadores personales obtenida en el ANOVA. Los profesores intuyen que estos programas, tal como están, no son adecuados para el desarrollo del potencial emprendedor. También coinciden en que el efecto medio alcanzado en el indicador orientación de logro obedece a la necesidad de recuperar el capital social invertido en las cooperativas para obtener beneficios en las ventas, alejándonos de cualquier otra interpretación posible de índole motivacional:

EFP3C11E: "Esos resultados confirman que los programas no están funcionando bien, ya lo intuíamos (...) se hace muy difícil sin programas, contenidos y evaluación concretos (...) se nos presupone una formación que no tenemos para el desarrollo de un proyecto asì».

Fayolle, Gailly y Lassas-Clerc (2006) plantean la conveniencia de la evaluación para obtener información sobre los procesos educativos en los PEE. Sin embargo, estos programas han carecido de procesos rigurosos evaluativos, y la visión del profesorado en relación con la implementación siempre ha quedado al margen, a diferencia de las sugerencias realizadas al respecto por Carey y Matlay (2011).

Compilando la información de las entrevistas y mediante una matriz dicotómica de presencia-ausencia de los ámbitos estudiados (Tabla 4), diríamos que este proyecto ha carecido de fundamentos teóricos que lo sustenten, así como de procesos evaluativos mínimamente significativos. Las finalidades y objetivos específicos fueron introduciéndose con el paso del tiempo, según la normativa emitida. Sin embargo, esas finalidades no correlacionaron con objetivos específicos congruentes, de dominio eminentemente empresarial o personal. Asimismo, los contenidos no están explícitos en ningún material educativo, al margen de que en las EM y EF se muestre la presencia de contenidos conceptuales de predominio empresarial, así como de habilidades sociales y de comunicación, pero sin 
suficiente congruencia interna. Por último, la metodología favorecedora del emprendimiento surgió a mitad del proyecto y se consolidó al final, mediante los métodos didácticos de aprendizaje cooperativo y de trabajo por proyectos.

Tabla 4

Matriz dicotómica ausencia-presencia de ámbitos de evaluación

\begin{tabular}{l|c|c|c|c|c|c|c|c|c|c|c|c|c|c|c}
\hline \multicolumn{10}{|c}{ ÁMBITOS DE } \\
EVALUACIÓN
\end{tabular}

A: ausencia de ámbitos de evaluación; $\mathrm{P}_{\mathrm{SCl}}$ presencia sin congruencia interna $\mathrm{NE}$ : profesor no entrevistado; $\mathrm{P}_{\mathrm{CCI}}$ : presencia con congruencia interna.

\section{CONCLUSIONES}

El efecto de este Proyecto en el potencial emprendedor del alumnado ha sido irrelevante. En lo referente a la primera hipótesis del estudio cuantitativo realizado, se concluye que la aplicación de los programas EME, ÍCARO y EJE no ha incrementado el nivel de las variables del potencial emprendedor (creatividad, liderazgo, resolución de problemas, control personal y puntuación global del test ATE), con excepción de la variable orientación al logro, con un tamaño de efecto medio. Respecto de la segunda hipótesis planteada, concluimos que el número de participaciones del alumnado no incide significativamente en la efectividad de los programas aplicados. Este pobre balance de resultados requiere alguna explicación.

Contrastando las argumentaciones del profesorado en las EF, atribuye el bajo impacto del proyecto sobre la actitud emprendedora a varias causas: falta de formación docente específica, incorrecto diseño de los programas, falta de planificación educativa para la implementación de EME, ÍCARO y EJE, carencia de contenidos e inexistencia de una correcta evaluación educativa. A juicio de los entrevistados, la ausencia de algunos elementos fundamentales en el diseño, junto con la falta de congruencia interna de otros, posibilitaría las bajas puntuaciones en el potencial emprendedor, así como la escasa valoración otorgada a la eficacia del proyecto. 
La práctica del profesorado es clave para determinar el logro de una cultura emprendedora en el sistema educativo. Pero una formación cultural y técnica que abunde en el sentido y alcance de una genuina educación emprendedora, inicial y continua, adquiere mayor relevancia, según los entrevistados. Línea de pensamiento que confirman diversos expertos (Jones e Iredale, 2010; Gibbs, 2011; Balan y Metcalfe, 2012).

Los PEE difieren en intensidad y posiblemente en eficacia. Pero algo que debe ser resuelto con premura es el ámbito de sus finalidades explícitas. Los profesores entrevistados coinciden en señalar cierta nebulosa perjudicial para la efectividad de los programas. Centrados en objetivos vinculados al pretendido desarrollo directo de la empresarialidad, apenas han dedicado atención a metas relacionadas con la dimensión más personal de la educación emprendedora. Esta únicamente se ha atendido, en algunos casos, para la mejora de la comunicación y la evitación del conflicto, en aras de facilitar el trabajo cooperativo perseguido. Esto podría explicar parcialmente el escaso desarrollo experimentado del potencial emprendedor.

Con visión retrospectiva, los profesores narran en las EF que, aun habiendo transcurrido cinco cursos, no ha existido un desarrollo de contenidos que evidencie qué habilidades, conocimientos y valores deben aprender los alumnos para potenciar adecuadamente su actitud emprendedora. Las narraciones desvelan un proyecto inclinado excesivamente hacia contenidos conceptuales y procedimentales vinculados con la gestión empresarial, mientras que los relacionados con valores y habilidades emprendedoras relativos al ámbito personal no han sido significativos.

Sobre la metodología empleada, de las narraciones podemos colegir las siguientes limitaciones: en primer lugar, faltan dinámicas con el alumnado que generen un trabajo cooperativo eficiente y eficaz (Negro, 2012), pues la mayoría de las veces los alumnos se agrupan por filiación, generándose controversias, disputas y falta de disciplina que dificultan el desarrollo adecuado de los programas. En segundo orden, aparece una excesiva inclinación hacia el cooperativismo como método de enseñanza, tal vez atenuada por la naturaleza misma del proyecto. Parece difícil considerar el cooperativismo como único elemento que genere actividad emprendedora (Puig y Martin, 2007). Por último, se encuentra la carencia metodológica relacionada con la capacidad de aumentar la motivación del alumnado hacia el proyecto, ya que parece disminuir paulatinamente cada curso.

La falta de información valorativa sobre el proceso de implementación ha constituido una deficiencia advertida por los docentes. Sin em- 
bargo, la complejidad de la educación emprendedora precisa de un trasvase múltiple de información entre alumnos, profesores, instituciones educativas y promotores (Jones y Matlay, 2011). Sin ese diálogo informativo entre las partes implicadas parece menoscabarse la correcta realización de los PEE (Matlay, 2006). De las EF se desprende que la imposibilidad de evaluar obedece al diseño del proyecto, ya que los programas no contienen información relevante para ser evaluada; de modo que se carece de datos concretos relacionados con aspectos metodológicos y de contenido del proyecto. Así, los programas se convierten en un fatal bucle donde la ausencia de los elementos constitutivos principales imposibilita su evaluación. A su vez, la no evaluabilidad del proyecto limita la mejora del diseño de los programas y su implementación.

Se precisa una fundamentación teórica sobre la educación emprendedora que facilite la labor profesoral (Pittaway, 2009). El diseño de los programas debe ser cuidadoso, puesto que de él depende su posible éxito. Reivindicado por los docentes, ha de configurarse con la suficiente flexibilidad y amplitud como para no excluir elementos necesarios del proceso formativo emprendedor (metas, métodos, contenidos, evaluación), especialmente aquellos relativos al mejor desarrollo posible de su potencial. Por tanto, el desarrollo de la capacidad de independencia y de iniciativa debería ser contemplado como un pilar básico. A través del despliegue de cualidades personales relacionadas con la identidad emprendedora es como se desarrolla el potencial emprendedor. Las deficiencias en el diseño de los programas estudiados parecen contener, entre otras, esta severa limitación.

Por último, todo debe estar vinculado a una planificación educativa adecuada, capaz de integrar la educación emprendedora eficientemente en el sistema educativo, lo que supone hallar respuestas de carácter integral y estructural. Posiblemente, convendría lograr un nuevo sentido del binomio contexto-educación, proponiendo cambios en los modos de participar y actuar por parte del alumnado y de otras instancias participantes. No podemos realizar determinados cambios hacia una "cultura educativa emprendedora» con los elementos y organización de una cultura tradicional y distinta.

Aunque precisemos extender estudios similares a muestras más amplias y a centros de diferentes características, para contrastar resultados y ampliar la discusión sobre la teoría y la práctica de los PEE, este estudio nos sugiere nuevas cuestiones susceptibles de investigación, a partir de la relevancia detectada de la dimensión personal de la identidad emprendedora: cómo abordar teórica y prácticamente la formación específica del profesorado; cómo integrar nuevos diseños con fórmulas no exclusi- 
vamente cooperativistas; qué conceptualización y modos organizativos podrían resultar integradores y exitosamente participativos; qué líneas metodológicas (incluyendo la retroalimentación evaluativa) alternativas y efectivas podríamos plantear; qué implicaciones y formas de conexión eficientes cabría esperar de una mayor vinculación entre la educación y el contexto emprendedor próximo; y, finalmente, qué modelos propedéuticos precisamos generar para la formación emprendedora en los escolares de las etapas obligatorias.

\section{NOTAS}

1 Financiación del proyecto EDU2013-42936-P del Ministerio de Economía y Competitividad, dentro del Plan Estatal de Excelencia de Proyectos I+D. 


\section{REFERENCIAS BIBLIOGRÁFICAS}

Alemany, L., Marina, J. A. y Pérez DíazPericles. J. M. (dir.) (2013). Aprender a emprender. Cómo educar el talento emprendedor. Barcelona: Fundación Príncipe de Girona/Aula Planeta.

Arnau, J. (1995). Diseños longitudinales aplicados a las ciencias sociales y del comportamiento. México D. F.: Noriega.

Athayde, R. (2009). Measuring enterprise potential in young people. Entrepreneurship Theory And Practice, 33(2), 481-500.

Athayde, R. (2012). The impact of enterprise education on attitudes to enterprise in young people: an evaluation study. Education + Training, 54(8/9), 709-726.

Balan, P. y Metcalfe, M. (2012). Identifying teaching methods that engage entrepreneurship Students. Education + Training, 54(5), 368-384.

Balluerka, N. y Vergara, A. I. (2002). Diseños de investigación experimental en psicología. Madrid: Prentice Hall.

Banister, P.; Burman, E.; Parker, I., Taylor, M. y Tindall, C. (2004). Métodos Cualitativos en Psicología: Una Guía Para la Investigación. Guadalajara: Universidad de Guadalajara.

Bericat, E. (1998). La integración de los métodos cuantitativo y cualitativo en la investigación social. Barcelona: Ariel.

Bernal, A. y Cárdenas, A. (2014). La formación de emprendedores en la escuela y su repercusión en el ámbito personal. Una investigación narrativa centrada en el Programa EME. Revista Española de Pedagogía, 257, 124-143.

Bisquerra, R. (2012). Metodología de la investigación educativa. Madrid: La Muralla.
Carey, C. \& Matlay, H. (2011). Emergent issues in Enterprise education: the educator's perspective. Industry and Higher Education, 25(6), 1-10.

Casanova, A. M. (2012). La evaluación de las competencias básicas. Madrid: La Muralla.

Chávez, E. G. (2012). EFL teachers' professional development within the educational ELT context at UNAN-León, Nicaragua. Alcalá de Henares: Servicio de Publicaciones de la Universidad de Alcalá.

Chell, E. (2008). The Entrepreneurial Personality: A Social Construction. Londres: Routledge.

COM (2012). Un nuevo concepto de educación: invertir en las competencias para lograr mejores resultados socioeconómicos. Estrasburgo: Comisión de las Comunidades Europeas 2012/669. Recuperado de http:// ec.europa.eu/education/news/rethinking/com669_es.pdf

Denzin, N. K. (1970). The Research Act. A Theoretical Introduction to Sociological Methods. Chicago: Aldine.

Draycott, M. C. \& Rae, D. (2011). Enterprise education in schools and the role of competency frameworks. International Journal of Entrepreneurial Behavior y Research, 17(2), 125-145.

Draycott, M. C., Rae, D. \& Vause, K. (2011). The assessment of enterprise education in the secondary education sector. A new approach? Education+training, 53(8/9), 673-681.

EACEA (2012). Entrepreneurship Education at School in Europe. National Strategies, Curricula and Learning Outcomes. Bruselas: Eurydice. 
Fayolle, A. \& Gailly, B. (2008). Teaching models and learning processes entrepreneurship education. Journal of European Industrial Training, 32 (7), 569-93.

Fayolle, A., Gailly, B. \& Lassas-Clerc, N. (2006). Assesing the impact of entrepreneurship education programmes: a new methodology. Journal of European Industrial, 30(9), 701-720.

Gewerc, A. y Montero, M. L. (2013). Culturas, formación y desarrollo profesional. La integración de las TIC en las instituciones educativas. Revista de Educación, 362, 323-347.

Gibbs, G. (2011). Concepts into practice: meeting the challenge of development of enterpreneurship educators around an innovative paradigm. International Journal of Enterpreneurial Behaviour and Research, 17(2), 146-165.

Gibbs, G. (2012). El análisis de datos cualitativos en investigación cualitativa. Madrid: Morata.

González, R. y Zúñiga, A. (2011). Método CEPCES para la evaluación del potencial emprendedor. Journal of Technology Management \& Innovation, 6(1), 77-99.

Habiby, A. S., Deirdre, M. y Coyle, J. (2010). El emprendedor de alta intensidad. Harvard Business Review, 88(8), 76-80.

Harmeling, S. S. (2011). Re-storying an entrepreneurial identity: education, experience and self-narrative. Education + Training, 53(8/9), 741-749.

Heyvaert, M., Hannes, K., Maes, B. \& Onghena, P. (2013). Critical Appraisal of Mixed Methods Studies. Journal of Mixed Methods Research March, 7(4), 302-327.

Johannisson, B. (1991). University training for entrepreneurship: a Swedish approach. Entrepreneurship and Regional Development, 3(1), 67-82.
Johnson, D. W., Johnson, R. T. \& Holubec, E. J. (1994). Cooperatiae Learning in the Classroom. Virginia: ASCD.

Jones, B. \& Iredale, N. (2010). Enterprise education as pedagogy. Education + training, 51(1), 7-19.

Jones, C. \& Matlay, H. (2011). Understanding the heterogeneity: of entrepreneurship education: going beyond Gartner. Education + Training, 53(8/9), 692-703.

Kvale, S. (2011). Las entrevistas de investigación cualitativa. Madrid: Morata.

León, O. G. y Montero, I. (2003). Métodos de investigación en Psicología y Educación. Madrid: McGraw-Hill.

Lieblich, A., Tuval-Mashiach, R. \& Zilber, T. (1998). Narrative research. Reading analysis, and interpretation. Thousand Oaks, CA: Sage.

Liñán, F., Santos, F. J. \& Fernández, J. (2011). The Influence of Perceptions on Potential Entrepreneurs. International Entrepreneurship and Management Journal, 7(3), 373-390.

Louise-Jayne, E. \& Muir, E. J. (2012). Evaluating enterprise education: why do it? Education + Training, 54(4), 278290.

Lumpkin, G. T. \& Dess, G. G. (1996). Clarifying the entrepreneurial orientation construct and linking it to performance. Academy of Management Review, 21(1), 135-172.

Maritz, A. \& Brown, C. R. (2013). Illuminating the black box of entrepreneurship. Education + Traininig, 55(3), 234-252.

Matlay, H. (2006). Researching entrepreneurship and education, part 2: what is entrepreneurship education and does it matter? Education + Training, 48(5), 577-597. 
Mayoh, J. \& Onwuegbuzie, A. J. (2015). Toward a Conceptualization of Mixed Methods Phenomenological Research. Journal of Mixed Methods Research, 9(1), 91-107.

Ministerio de Industria, Turismo y Comercio (2010). Panel informativo $n .^{\circ}$ 27. Madrid: Dirección General de Política de la pequeña y mediana empresa.

Morgan, D. L. (1998). Practical strategies for combining qualitative and quantitative methods: Applications to health research. Qualitative Health Researchs, 8(3), 362-376.

Nabi, G. y Liñán, F. M. (2011). Graduate entrepreneurship in developing countries: intentions, education and development. Education + Training, 53(5), 325-334.

Negro, A. (2012). Aprendizaje cooperativo en las aulas: fundamentos y recursos para su implantación. Madrid: Alianza Editorial.

Pardo, A., Garrido, J., Ruiz, M. A. y San Martín, R. (2007). La interacción entre factores en el análisis de varianza: errores de interpretación. Psicothema, 19(22), 343-349.

Pérez Juste, R. (2006). Evaluación de Programas Educativos. Madrid: La Muralla.

Pérez Serrano, G. (1994). Investigación cualitativa. Retos e interrogantes. II. Técnicas y análisis de datos. Madrid: La Muralla.

Pittaway, L. (2009). The role of inquirybased learning in entrepreneurship education. Industry and Higher Education, 23(3), 153-62.

Puig, J. M. y Martin, X. (2007). Competencia en autonomía e iniciativa personal. Madrid: Alianza Editorial.
Rae, D. y Woodier-Harris, N. (2012). International entrepreneurship education: Postgraduate business student experiences of entrepreneurship education. Education + Training, 54(8/9), 639-656.

Railsback, J. (2002). Project-based instruction: Creating excitement for learning. Portland, OR: Northwest Regional Educational Laboratory.

Roca, E. (2013). La evaluación diagnóstica de las competencias básicas. Madrid: Síntesis.

Ruiz, J. I. (2009). Metodología de la investigación cualitativa. Bilbao: Universidad de Deusto.

Ruskovaara, E. \& Pihkala, T. (2013). Teachers implementing entrepreneurship education: classroom practices. Education + training, 55(2), 204-216.

Seikkula-Leino, J., Ruskovaara, E., Ikävalko, M., Mattila, J. \& Rytkola, T. (2010). Promoting entrepreneurship education: the role of the teacher? Education + training, 52(2), 117-127.

Steenekamp, A. G., van der Merwe, S. P. \& Athayde, R. (2011). An investigation into youth entrepreneurship in selected South African secondary schools: an exploratory study. Southern African Business Review, 15(3), 46-75.

Tójar, J. C. (2006). Investigación cualitativa. Comprender y actuar. Madrid: La Muralla.

Vesa, T. \& Down, S. (2012). Measuring entrepreneurial orientation of university students. Education + Training, 54(8/9), 744-760.

Yin, R. K. (2009). Case Study Research: Design and Methods. Thousand Oaks, CA: Sage. 


\section{PERFIL ACADÉMICO Y PROFESIONAL DE LOS AUTORES}

Antonio Bernal Guerrero. Catedrático de Teoría de la Educación. Departamento de Teoría e Historia de la Educación y Pedagogía Social, de la Universidad de Sevilla. Doctor en Filosofía y Ciencias de la Educación, con Premio Extraordinario. Dirige el Grupo de Investigación Pedagógica de la Persona en la misma Universidad. Autor de numerosas publicaciones. Actualmente, dirige una investigación sobre educación emprendedora, en el marco de los proyectos nacionales de excelencia.

Antonio Ramón Cárdenas Gutiérrez. Doctor en Pedagogía por la Universidad de Sevilla, con Premio Extraordinario. Profesor Asociado de la Facultad de Ciencias de la Educación de la Universidad de Sevilla (acreditado a Profesor Contratado Doctor) y orientador. Miembro del Grupo de Investigación Pedagógica de la Persona. Sus trabajos recientes se centran en la construcción de la identidad, concretamente en el ámbito de la emprendeduría.

Dirección de los autores: Facultad de Ciencias de la Educación Universidad de Sevilla

C/ Pirotecnia, s/n

41013 Sevilla

E-mail: abernal@us.es acardenas1@us.es

Fecha Recepción del Artículo: 02. Marzo. 2015

Fecha modificación Artículo: 03. Mayo. 2015

Fecha Aceptación del Artículo: 12. Junio. 2015

Fecha Revisión para Publicación: 09. Marzo. 2017 